Imaging the early material response associated with exit surface damage in fused silica

S. G. Demos, R. N. Raman, R. A Negres

November 15, 2010

SPIE Laser Damage Conference

Boulder, CO, United States

September 26, 2010 through September 29, 2010 
This document was prepared as an account of work sponsored by an agency of the United States government. Neither the United States government nor Lawrence Livermore National Security, LLC, nor any of their employees makes any warranty, expressed or implied, or assumes any legal liability or responsibility for the accuracy, completeness, or usefulness of any information, apparatus, product, or process disclosed, or represents that its use would not infringe privately owned rights. Reference herein to any specific commercial product, process, or service by trade name, trademark, manufacturer, or otherwise does not necessarily constitute or imply its endorsement, recommendation, or favoring by the United States government or Lawrence Livermore National Security, LLC. The views and opinions of authors expressed herein do not necessarily state or reflect those of the United States government or Lawrence Livermore National Security, LLC, and shall not be used for advertising or product endorsement purposes. 


\title{
Imaging the early material response associated with exit surface damage in fused silica
}

\author{
Stavros G. Demos*, Rajesh N. Raman, Raluca A. Negres \\ Lawrence Livermore National Laboratory, 7000 East Avenue, Livermore, CA, USA 94550
}

\begin{abstract}
The processes involved at the onset of damage initiation on the surface of fused silica have been a topic of extensive discussion and thought for more than four decades. Limited experimental results have helped develop models covering specific aspects of the process. In this work we present the results of an experimental study aiming at imaging the material response from the onset of the observation of material modification during exposure to the laser pulse through the time point at which material ejection begins. The system involves damage initiation using a $355 \mathrm{~nm}$ pulse, $7.8 \mathrm{~ns}$ FWHM in duration and imaging of the affected material volume with spatial resolution on the order of $1 \mu \mathrm{m}$ using as strobe light a $150 \mathrm{ps}$ laser pulse that is appropriately timed with respect to the pump pulse. The observations reveal that the onset of material modification is associated with regions of increased absorption, i.e., formation of an electronic excitation, leading to a reduction in the probe transmission to only a few percent within a time interval of about $1 \mathrm{~ns}$. This area is subsequently rapidly expanding with a speed of about $1.2 \mu \mathrm{m} / \mathrm{ns}$ and is accompanied by the formation and propagation of radial cracks. These cracks appear to initiate about $2 \mathrm{~ns}$ after the start of the expansion of the modified region. The damage sites continue to grow for about $25 \mathrm{~ns}$ but the mechanism of expansion after the termination of the laser pulse is via formation and propagation of lateral cracks. During this time, the affected area of the surface appears to expand forming a bulge of about $40 \mu \mathrm{m}$ in height. The first clear observation of material cluster ejection is noted at about 50 ns delay.
\end{abstract}

Keywords: Time-resolved imaging, microscopy, fused silica, surface damage initiation, damage growth

\section{INTRODUCTION}

ICF class laser systems utilize large aperture optical components that are exposed to relatively high laser fluences which, however, are still about one order of magnitude below the intrinsic damage threshold of the material used. Under these excitation conditions, laser-induced damage in the optical components of the laser system originates in the presence of some type of defect that leads to coupling of the laser energy into the material. The exit-surface damage initiation and growth in fused silica optics has been a problem that scientists and engineers working in the development of ICF class laser system have devoted considerable attention. This is due to the fact that damage initiation can lead to catastrophic failure of the material due to the growth of the original damage site(s) with subsequent exposure to a relatively small number of laser pulses. Damage initiation sites are typically on the order of $10 \mu \mathrm{m}$ in diameter, depending on the laser pulse length, but tend to grow exponentially under subsequent ns laser irradiation [1-6]. Microscopic examination of these sites reveals the formation of a damage crater containing modified material believed to be the result of exposure to high pressures and/or temperatures, as well as cleaved surfaces and cracks [6-10]. Although various models regarding the processes involved during a surface damage event have been proposed based on post-mortem examination of the crater characteristics for various laser parameters, there are only limited experimental studies that capture the dynamic processes during a damage event [11-13]. Of particular importance are experimental data on the laser-material interactions during the laser pulse that governs the energy deposition process. It is therefore apparent that an imaging system capable of capturing the material response through the timeline of events involved during surface damage would greatly enhance our understanding of the fundamental mechanisms involved.

In previous work we discussed the results using a time resolved imaging system that was employing a 7-ns in time duration (at full width at half maximum, FWHM) laser (Quanta-Ray, Spectra-Physics) operating at $355 \mathrm{~nm}(3 \omega)$ to initiate damage on the exit surface of the fused silica optic [14-15]. The images were captured using strobe-light illumination provided by a $532 \mathrm{~nm}(2 \omega), \sim 4.5 \mathrm{~ns}$ (at FWHM) probe pulse (Litron Lasers, UK). The system was capable of acquiring two transient images per each damage event using strobe pulses obtained by two separate lasers with output polarizations orthogonal with respect to each other. To separate the images from each probe arriving at a different delay,

*demos1 @1lnl.gov; phone 1925 423-3388 
we utilized a polarization filtering method in which the two probe beams were first combined into a single beam path using a polarizing beam-splitter and the image components from each probe were subsequently separated and directed to separate CCD cameras using a polarizing beam-splitter located behind the microscope's image-forming optics. The timing of the probe pulses was controlled by adjustable delay trigger in reference to the pump pulse, allowing for image acquisition at any time point covering the entire timeline of events during damage initiation or growth. The arrival of the pump and probe pulses were separately recorded for each measurement using a set of fast photodiodes and the peak-topeak time delay was obtained with $\sim 0.5 \mathrm{~ns}$ resolution using a $500 \mathrm{MHz}$ oscilloscope.

This system was proven successful in capturing the kinetics of the ejected material but the preliminary results indicated that the material transformation during the early phase (when the pump pulse is on) was occurring much faster than the temporal resolution provided by the $4.5 \mathrm{~ns}$-duration probe pulses [15]. Consequently, a more advanced system was required to capture the characteristic processes at early times with adequate spatial and temporal resolution. The effort to develop a more suitable system to study the early material response associated with exit surface damage in fused silica along with preliminary results are discussed in this work. Specifically, after presenting in detail our experimental system that enabled this study, we will summarize our preliminary interpretation of the experimental results.

\section{EXPERIMENTAL SYSTEM}

The samples used in this study were circular fused silica optical flats having diameter of $50 \mathrm{~mm}$ and thickness of $10 \mathrm{~mm}$. To simulate damage occurring under operating conditions relevant to large-aperture laser systems, the damaging (or ритр) laser pulse must spatially overfill the initiated or growing damage sites, which can be on the order of $10 \mu \mathrm{m}$ or $100 \mu \mathrm{m}$ in diameter, respectively. To meet this requirement, a 150-mm focal length lens was used to focus the output of the pump laser (Quanta-Ray, Spectra-Physics) operating at $355 \mathrm{~nm}(3 \omega), 7-\mathrm{ns}$ FWHM in duration, about $20 \mathrm{~mm}$ behind the exit-surface of the sample. In this arrangement, we estimate the pump beam diameter at the exit surface to be $\sim 1 \mathrm{~mm}$. Pristine surface sites were exposed to an average fluence of $\sim 50 \mathrm{~J} / \mathrm{cm}^{2}$ and damage initiation was observed with $\sim 80 \%$ probability. This procedure leads to the formation of single or multi-pit craters with average pit diameter on the order of $10-50 \mu \mathrm{m}$.

The configuration of the imaging system remains practically identical to that previously discussed [14-15] involving two microscope systems positioned parallel and perpendicular to the sample's surface. The corresponding images are referred to as the transmission-view (TV) and side-view (SV) images, respectively. Both microscopes were identical, utilizing a long working distance $5 \mathrm{X}$ objective (Mitutoyo, Japan) followed by a $5 \mathrm{X}$ zoom lens. The images were captured by a CCD detector (Spiricon, 1616 pixels $\times 1216$ pixels) with individual pixel size of $4.4 \mu \mathrm{m}$. The resulting spatial resolution was on the order of $1 \mu \mathrm{m}$. The focal planes of the two microscopes intersected at the sample's surface at the pump beam location. In this way, some of the damage sites formed in this volume could be imaged with optimal spatial resolution by both microscopes.

A limitation in temporal resolution resulted in loss of the spatial resolution of the images acquired at early delay times. Specifically, key aspects of the damage process include a) the transient phases of the material response, b) the initial phase of the shock front in air and in the material, c) the early motion of the surface and, d) the early kinetics of the fast ejected particles. For example, the speed of the pressure front generated following laser energy deposition in fused silica is equal or higher to the speed of sound in the material, or $\sim 6 \mu \mathrm{m} / \mathrm{ns}$. In this case, the $\sim 4.5 \mathrm{~ns}$ probe pulse could only provide spatial resolution on the order of $30 \mu \mathrm{m}$ when capturing these events. To resolve the dynamics of the above key parameters within the $\approx 1 \mu \mathrm{m}$ spatial resolution of the imaging system, the pulse length of the laser should be sufficiently short. For example, to resolve the pressure front with $1 \mu \mathrm{m}$ spatial resolution, the probe pulse length should be about 170 ps. Furthermore, the imaging system must be able to capture two images per each event spaced closely in time (subnanosecond) in order to better estimate instantaneous speed as this may be changing during the timeline.

To meet these requirements, the probe lasers $4.5 \mathrm{~ns}$ in pulse duration described in Ref. 15 were replaced with a single laser having a pulse length of $\sim 150 \mathrm{ps}$ (EKSPLA, Lithuania). To allow for imaging of two time points per damage event, two probe pulses (probe 1 and probe 2) were generated by splitting the output from the $150 \mathrm{ps}$ laser into two paths using a non-polarizing beam-splitter, as shown in Fig. 1. The reflected beam (probe 2) was directed towards an adjustable delay line with $\sim 60 \mathrm{~cm}$ roundtrip, thus providing a relative delay between probe 1 and probe 2 of up to $\sim 4.15 \mathrm{~ns}$. A halfwave plate was inserted within the delay line in order to rotate the polarization of probe 2 to a state orthogonal to that of probe 1 (to allow for polarization filtering as described in Ref. 15). Both probe beams were then recombined using a polarizing beam-splitter and followed a common path up to a second non-polarizing beam-splitter. The latter element 
provided SV and TV paths to illuminate the sample. Finally, a polarizing beam-splitter positioned after the TV image formation optics separated the two orthogonal polarization image components (delayed with respect to each other) and directed them onto separate cameras (Fig. 1, CCDs 1-2). The temporal profiles of the pump and probe pulses were recorded using a high-speed GaAs photodetector (rise time <35 ps, Electro-Optics Technology, Inc.) and digital oscilloscope (Tektronix) with sampling rate capability of 25 Gsamples/s.

The SV mode of the system shown in Fig. 1 can also be used to image the material transformation occurring within the bulk material underneath the surface with high temporal resolution. Imaging through the side of the sample required flat and well polished sides with optical quality finish to transmit the probe beam for SV shadowgraphic image acquisition. The focal plane of the imaging optics was adjusted to coincide with the crater location while imaged within the bulk of the material. We note that material motion in the air-side of the interface is no longer visible in this configuration due to the optical path difference when imaging through the bulk compared to that when imaging through the air-side.

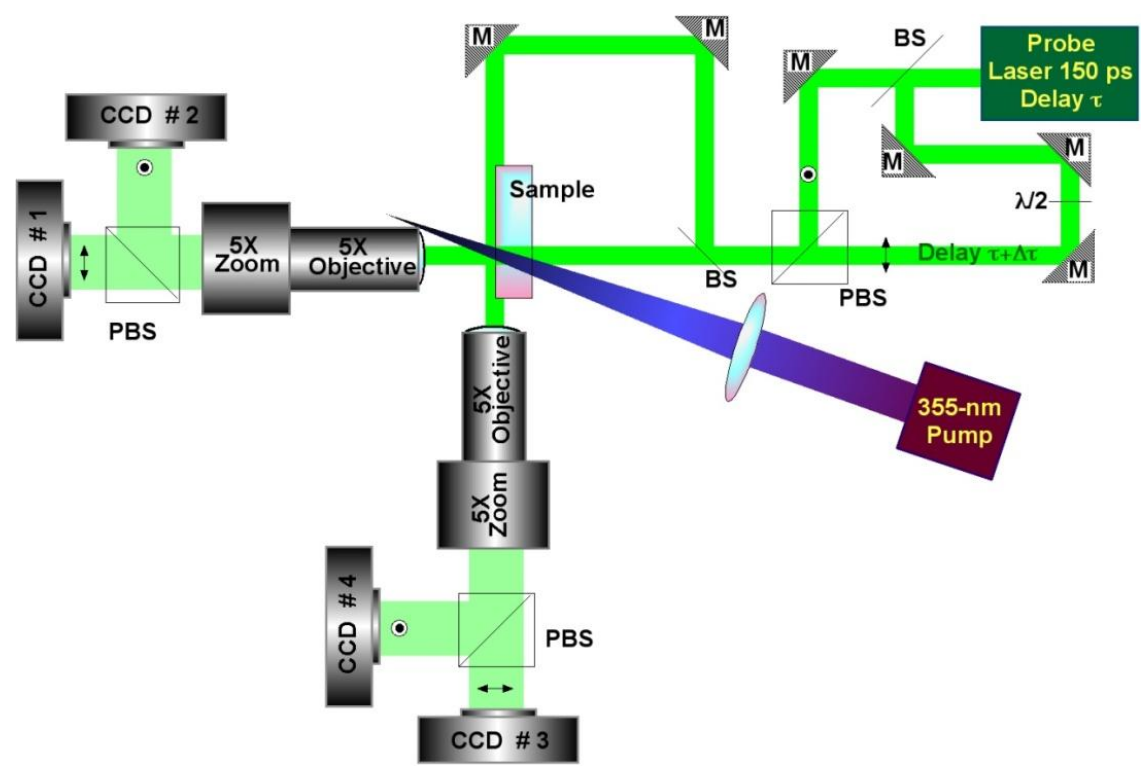

Figure 1. Experimental layout of time-resolved microscope system for simultaneous acquisition of transmissionview (TV) and side-view (SV) images with high temporal resolution (150 ps) using a dual probe imaging configuration with polarization filtering.

\section{PRELIMINARY RESULTS}

Typical images during the early stages of the damage initiation event acquired in the TV mode with this higher temporal resolution system are shown in Fig. 2. Such images reveal the material response during energy deposition by the pump pulse. The image shown in Fig. 2a was obtained from probe 1 pulse which arrived at the imaged area (damage site) $\sim 0.7$ $\mathrm{ns}$ before the peak of the pump pulse (defined as delay $\mathrm{t}=0$ ). Similarly, the image shown in Fig. $2 \mathrm{~b}$ represents the evolution of the damage site $1.19 \mathrm{~ns}$ after the image shown in Fig. 2a was captured (or t $~ 0.46 \mathrm{~ns}$ delay). From these images, one can easily appreciate that the material begins to optically absorb early on during the damage initiation process and these absorbing regions grow in size during exposure to the laser pulse. The final dimensions of the damage craters are much larger as can be seen in Fig. 2(c).

The images captured during the time the pump laser is on (for delays up to about $8 \mathrm{~ns}$ delay) demonstrated an initial build up of an electronic excitation on the surface which is manifested as a loss in transmission in the TV mode. Thereafter, some of the localized absorbing regions start growing with a lateral speed on the order of $1-2 \mathrm{Km} / \mathrm{sec}$. At later delays (but still while the pump pulse is on) radial cracks are observed to initiate and grow. The region of modified material continues to expand after the pump pulse is turned off up to a delay of about $25 \mathrm{~ns}$. 

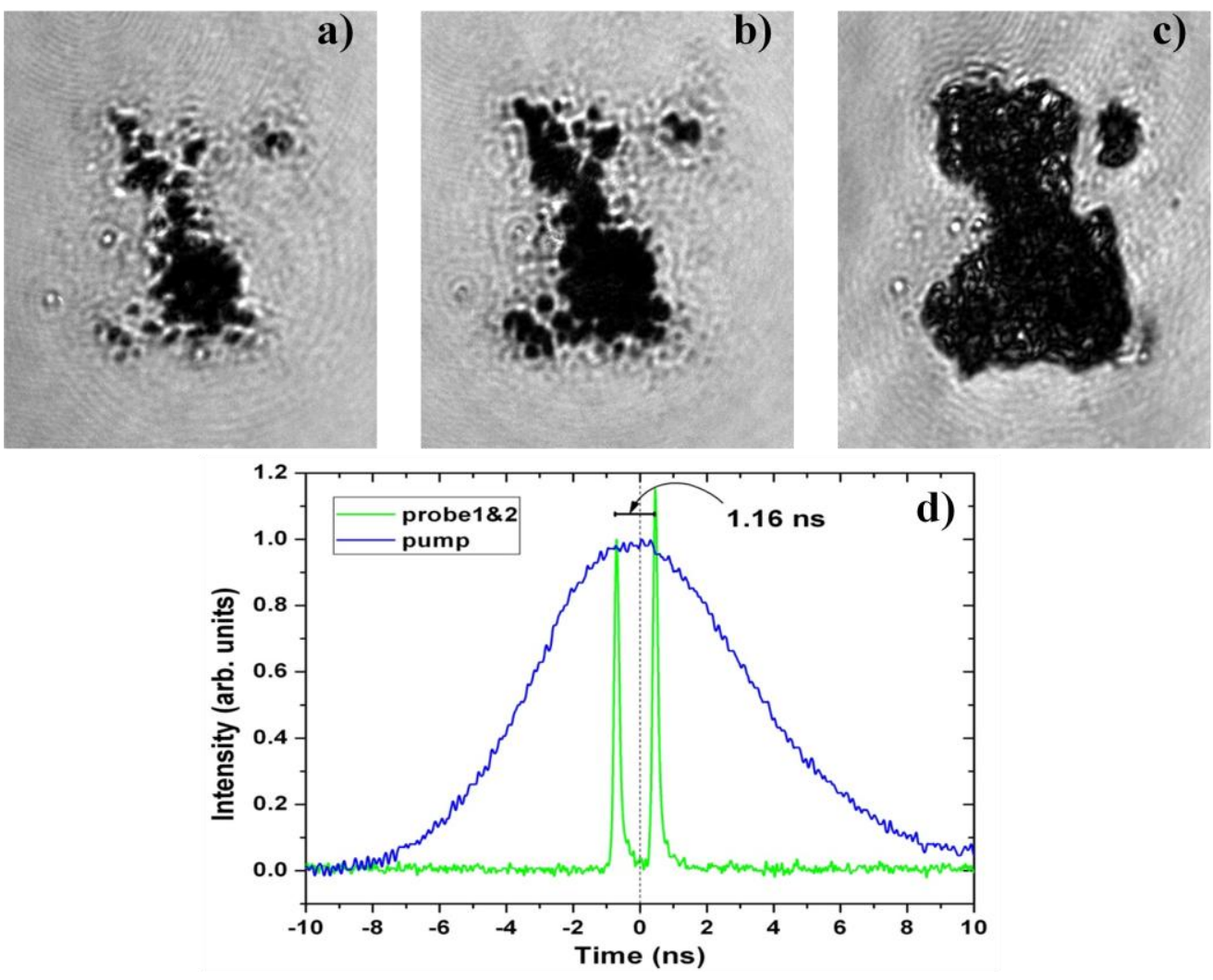

Figure 2. Typical high temporal resolution TV images of the same damage initiation event. Images captured shortly (a) before and (b) after arrival of the peak of the pump pulse; (c) final state of the damage site. Images are $163 \mu \mathrm{m} \times 181 \mu \mathrm{m}$. (d) Plot of temporal profiles and relative arrival times of the pump, probe 1 , and probe 2 pulses for the event illustrated in $(\mathrm{a}-\mathrm{c})$. Probe delay is defined with respect to the peak of the pump pulse $(\mathrm{t}=0)$.

The system shown in Fig. 1 can also be used to image the transient stress fields generated by the propagating pressure (shock) wave. This is achieved by blocking the path of probe 2 and utilizing only probe 1 . The polarizing beam-splitter following the TV imaging optics can be used to separate the orthogonal image components (parallel and perpendicular) and direct them to separate CCD's. Stress fields will rotate the polarization of the propagating light and a projection of their spatial distribution can be captured in the cross-polarized camera.

Figure 3 shows examples of polarization-dependent transient TV images taken at $\mathrm{t} \sim 13.4 \mathrm{~ns}$ delay. The parallel polarization image (Fig. 3(a)) represents the conventional shadowgraphy image showing the region of the material that has been modified as well as the location of the shock in the air side of the interface. In contrast, the orthogonal polarization image maps the stress fields associated with shock front propagation in the material, as shown in Fig. 3(b). In the final images (not shown), residual stress was only observed near where cracks had been formed. This configuration is particularly useful for estimating the kinetics of the shock waves in the bulk of the material.

The ability of this system to image the evolution of the material modifications in the bulk during an exit surface damage process is demonstrated in Fig. 4 where raw transient and final images from two different damage sites are shown. The observed vertical, fine fringe pattern is a result of interference caused by partial reflection of the probe beam on the sample surface and can be removed with image processing. Comparison of the transient (a, b at $5.6 \mathrm{~ns}$ and $8.1 \mathrm{~ns}$ probe delay, respectively) with the corresponding final images (c,d) shown in Figure illustrate the ability of the system to capture the dynamics of the expansion of the modified region beneath the surface. Transient features such as shock front propagation, crater expansion in both lateral and axial directions, and crack growth into the bulk are captured and reveal 
that the crater is evolving over this time scale. The final images of these sites are shown in Figs. 4(c-d) for reference. Similar to the dual-probe TV imaging approach capturing two time points per event (Fig. 2), this system can also be used to infer the kinetics of the material modifications at early times. Finally, high-resolution imaging of shock fronts and the material motion at early delay times in the air-side of the surface can also be performed using the short pulse-length laser with adequate spatial and temporal resolution.
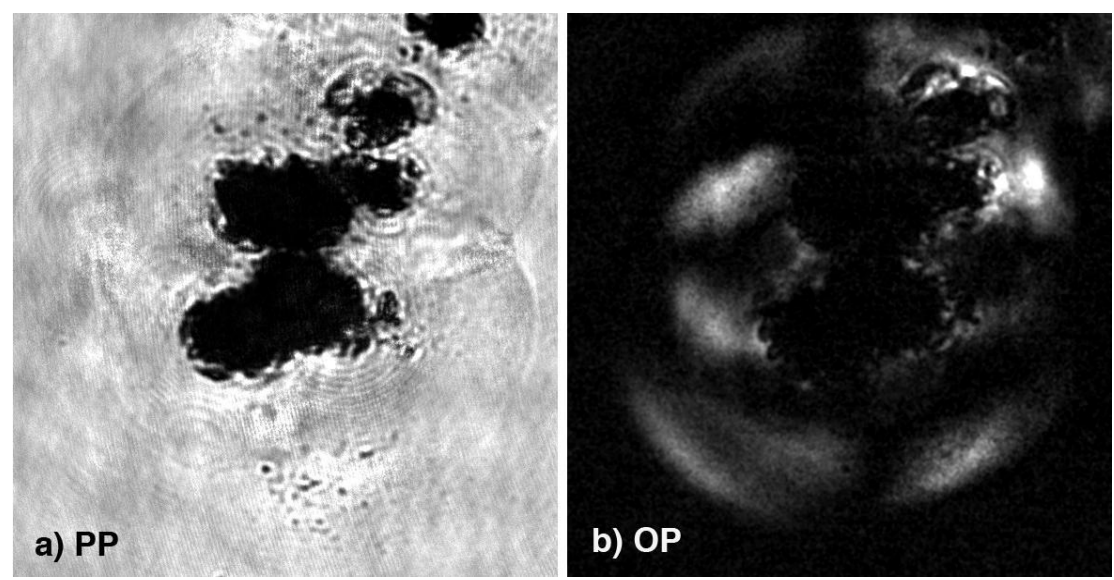

Figure 3. Example of high temporal resolution, polarization-sensitive images taken simultaneously in the TV mode $13.4 \mathrm{~ns}$ delay after the peak of the pump pulse. (a) $\mathrm{PP}=$ parallel polarization image component and (b) $\mathrm{OP}=$ orthogonal polarization image component, relative to that of the incident probe pulse. Image size is $290 \mu \mathrm{m} \times 305 \mu \mathrm{m}$.

Site 1

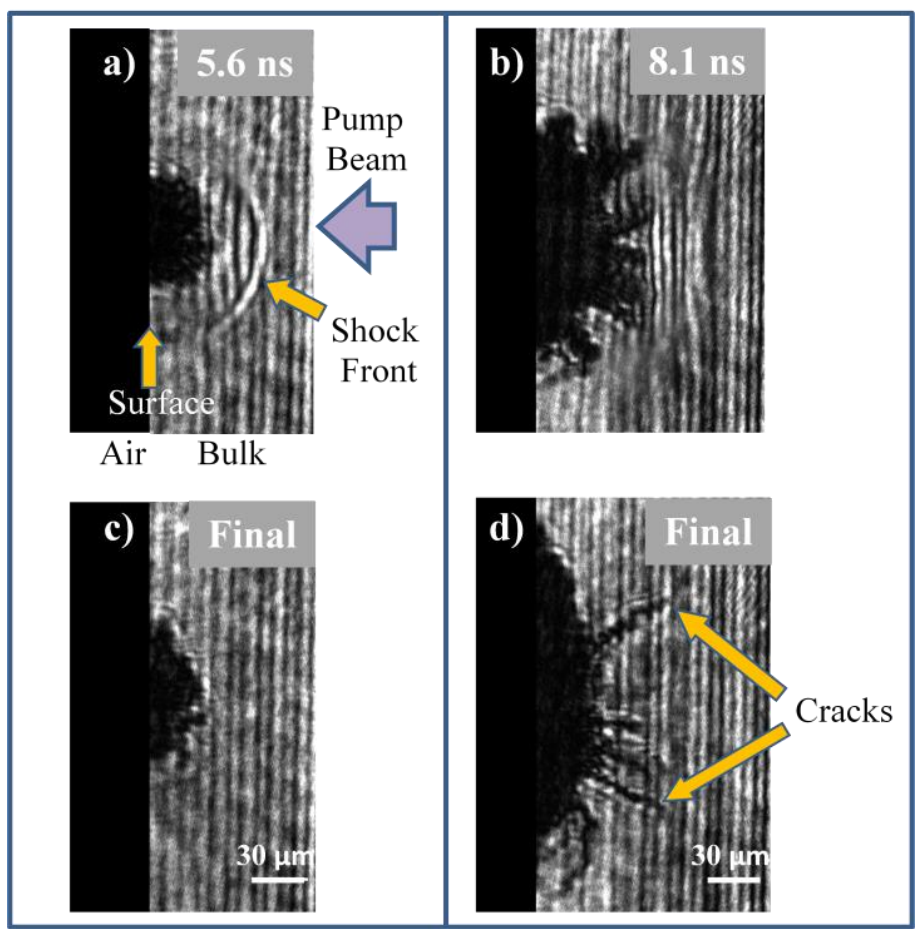

Figure 4. a-b) Typical SV images of bulk transient modifications from two different damage sites at the specified delay times. (c-d) The corresponding final images. 
SV images obtained from the air side of the sample are suggestive that there is surface swelling in the affected locations on the surface of the material leading to the formation of a bulge. The material swelling on the surface precedes the ejection of individual particles. This observation reveals a critical part of the early material response to localized energy deposition. The ejection process starts at about 25-35 ns delay with the ejection of small particles having diameter on the order of $1 \mu \mathrm{m}$ and speeds on the order of $2-3 \mathrm{Km} / \mathrm{sec}$. A jet of material ejecta is observed at longer delays.

\section{DISCUSSION}

The experimental results from this and the previous $[14,15]$ study indicate that the material response during a laser damage event is associated with a series of complex processes that current modeling tools fail to accurately describe and reproduce. Although it is not the intention of this work to provide a detailed description of the experimental observations, the preliminary findings regarding exit surface damage in fused silica optical components using 7-ns FWHM, $355 \mathrm{~nm}$ laser pulses can be summarized as follows:

- Onset of electronic excitation, manifested as a reduction in transmission of light through affected regions

- Lateral expansion of the modified region along the surface for about $25 \mathrm{~ns}$. The initially symmetrically expanding modified region is followed by the onset and growth of radial cracks and subsequently by lateral cracks

- Axial expansion of the modified region in the bulk for about $3 \mathrm{~ns}$ accompanied by the onset and growth of radial cracks during the later part of this phase

- Swelling of the surface until about $25 \mathrm{~ns}$ delay leading to the formation of a bulge

- Ejection of small material clusters (1-2 $\mu \mathrm{m}$ in diameter) starting at about 25-35 ns delay having speeds on the order of $2.5 \mathrm{Km} / \mathrm{sec}$

- Collapse of the bulge at about 50-75 ns delay followed by the formation of a jet of particles 1-30 $\mu \mathrm{m}$ in diameter and variable speeds ranging from about $2.5 \mathrm{Km} / \mathrm{sec}$ down to about $10 \mathrm{~m} / \mathrm{sec}$

- Between 100 and 2000 ns delay, a jet of nearly spherical or irregularly shaped clusters is formed with diameters between 1-25 $\mu \mathrm{m}$. Ejection of material at later times includes flakes and chips with diameters up to $50 \mu \mathrm{m}$ or larger

- Beyond $2 \mu$ s delay, the ejection of material clusters continues while the ejecta speed continues to decrease to about $10 \mathrm{~m} / \mathrm{sec}$. Termination of the ejection process appears to take place at $\sim 4 \mu$ s delay for damage initiation

Similar experiments performed during damage growth are strongly suggestive that damage initiation and growth evolve in the same manner but with two characteristic differences: a) the duration of the material ejection process, as discussed above and b) the speed of the shock front in the air, which is higher for damage growth, indicative of a higher energy deposition event. Furthermore, the time resolved images at the onset of damage growth suggest that damage growth is associated with multiple initiation events located in close proximity to each other, with the separation distance on average depending on laser excitation conditions (such as fluence).

\section{CONCLUSIONS}

Using a versatile time-resolved microscope system, we were able to obtain, for the first time, detailed quantitative information on the damage timeline. We have determined that the entire timeline of damage initiation on the exit surface of fused silica under exposure to high power ns pulses is about $4 \mu \mathrm{sec}$. We were able to identify the individual processes and their role in the final morphology of the damage site which can depend on the laser pulse parameters. These experimental results can be used to validate models and hydro-codes for use to predict damage behavior in optical materials for ICF class laser systems. We postulate that development of fundamental understanding of laser induced damage will help advance our broader knowledge regarding the material response when exposed to extreme conditions especially when the energy delivery is performed locally with a high power laser pulse (such as in laser ablation and laser micro-machining). 


\section{ACKNOWLEDGEMENTS}

This work was performed under the auspices of the U.S. Department of Energy by Lawrence Livermore National Laboratory under Contract DE-AC52-07NA27344.

\section{REFERENCES}

[1] Norton, M. A., Hrubresh, L. W., Wu, Z. L., Donohue, E. E., Feit, M. D., Kozlowski, M. R., Milam, D., Neeb, K. P., Molander, W. A., Rubenchik, A. M., Sell, W. D., and Wegner, P., "Growth of laser initiated damage in fused silica at $351 \mathrm{~nm}, "$ Proc. SPIE 4347, 468-468 (2001).

[2] Razè, G., Morchain, J. -M., Loiseau, M., Lamaignère, L., Josse, M., and Bercegol, H., "Parametric study of the growth of damage sites on the rear surface of fused silica windows," Proc. SPIE 4932, 127-135 (2003).

[3] Norton, M. A., Adams, J. J., Carr, C. W., Donohue, E. E., Feit, M. D., Hackel, R. P., Hollingsworth, W. G., Jarboe, J. A., Matthews, M. J., Rubenchik, A. M., and Spaeth, M. L., "Growth of laser damage in fused silica: diameter to depth ratio," Proc. SPIE 6720, 67200H (2007).

[4] Carr, C. W., Trenholme, J. B., and Spaeth, M. L., "Effect of temporal pulse shape on optical damage," Appl. Phys. Lett. 90(4), 041110 (2007).

[5] Huang, W. Q., Han, W., Wang, F., Xiang, Y., Li, F. Q., Feng, B., Jing, F., Wei, X. F., Zheng, W. G., and Zhang, X. M., "Laser -induced damage growth on large aperture fused silica optical components at $351 \mathrm{~nm}$," Chin. Phys. Lett. 26(1), 017901 (2009).

[6] Negres, R. A., Norton, M. A, Cross, D. A., Carr, C. W., "Growth behavior of laser-induced damage on fused silica optics under UV, ns laser irradiation,”Opt. Express, 18 (19), 19966-19976 (2010).

[7] Kucheyev, S. O. and Demos, S. G., "Optical defects produced in fused silica during laser-induced breakdown," Appl. Phys. Lett. 82(19), 3230-3232 (2003).

[8] Wong, J., Ferriera, J. L., Lindsey, E. F., Haupt, D. L., Hutcheon, I. D., and Kinney, J. H., "Morphology and microstructure in fused silica induced by high fluence ultraviolet $3 \omega(355 \mathrm{~nm})$ laser pulses," J. Non-Cryst. Solids 352(3), 255-272 (2006).

[9] Demos, S. G., Staggs, M., Minoshima, K., and Fujimoto, J., "Characterization of laser induced damage sites in optical components" Opt. Express 10(25), 1444-1450 (2002).

[10] Negres, R. A., Burke, M. W., DeMange, P. P., Sutton, S. B., Feit, M. D., and Demos, S. G., "Evaluation of UV absorption coefficient in laser-modified fused silica," Appl. Phys. 90(6), 061115 (2007).

[11] Russo, R. E., Mao, X. L., Liu, H. C., Yoo, J. H., and Mao, S. S., "Time-resolved plasma diagnostics and mass removal during single-pulse laser ablation," Appl. Phys. A-Mater. 69, S887-S894 (1999).

[12] Carr, C. W., Radousky, H. B., Rubenchik, A. M., Feit, M .D., and Demos, S. G., "Localized dynamics during laserinduced damage in optical materials," Phys. Rev. Lett. 92(8), 087401 (2004).

[13] Negres, R. A., Feit, M. D., DeMange, P., Bude, J. D., and Demos, S. G., "Pump and probe damage testing for investigation of transient material modifications associated with laser damage in optical materials," Proc. SPIE 6720, 672019-1-6 (2008).

[14] Stavros G. Demos, Rajesh N. Raman, Raluca A. Negres, "Time-resolved imaging of material cluster ejection during exit-surface damage initiation and growth in fused silica", 41st Annual Laser Damage Symposium, Laser-Induced Damage in Optical Materials: 2009, 21-23 September 2009, Boulder, Colorado,USA

[15] Raman, R. N., Negres, R. A., Demos, S. G., "Imaging system to measure kinetics of material cluster ejection during exit-surface damage initiation and growth in fused silica," Proc. SPIE 7504, 750418 (2009). 